\title{
MEASURE THEORETICAL ENTROPY OF COVERS
}

\author{
URI SHAPIRA
}

\begin{abstract}
In this paper we introduce three notions of measure theoretical entropy of a measurable cover $\mathcal{U}$ in a measure theoretical dynamical system. Two of them were already introduced in $[R]$ and the new one is defined only in the ergodic case. We then prove that these three notions coincide, thus answering a question posed in $[R]$ and recover a variational inequality (proved in $[\mathrm{GW}]$ ) and a proof of the classical variational principle based on a comparison between the entropies of covers and partitions.
\end{abstract}

\section{INTRODUCTION}

In this paper a measure theoretical dynamical system (m.t.d.s) is a four tuple $(X, \mathcal{B}, \mu, T)$, where $(X, \mathcal{B})$ is a standard space (i.e isomorphic to $[0,1]$ with the Borel $\sigma$-algebra, $\mu$ is a probability measure on $(X, \mathcal{B})$ and $T$ is an invertible measure preserving map from $X$ to itself.

A topological dynamical system (t.d.s) is a pair $(X, T)$, where $X$ is a compact metric space and $T$ is a homeomorphism from $X$ to itself.

In $[R]$ the author introduced two notions of measure theoretical entropy of a cover, both generalizing the definition of measure theoretical entropy of a partition and influenced by [BGH]. Namely,

(1) $h_{\mu}^{+}(\mathcal{U})=i n f_{\alpha \succeq \mathcal{U}} h_{\mu}(\alpha)$

(2) $h_{\mu}^{-}(\mathcal{U})=\lim \frac{1}{n} \inf f_{\alpha \succeq \mathcal{U}_{0}^{n-1}} H_{\mu}(\alpha)$

It was shown there among other things that $h_{\mu}^{-}(\mathcal{U}) \leq h_{\mu}^{+}(\mathcal{U})$ and that in the topological case (i.e a t.d.s and an open cover), one can always find an invariant measure $\mu$ such that $h_{\mu}^{-}(\mathcal{U})=h_{\text {top }}(\mathcal{U})$. This generalizes the result from $[\mathrm{BGH}]$ asserting that in the topological case one can always find an invariant measure $\mu$ such that $h_{\mu}^{+}(\mathcal{U}) \geq h_{\text {top }}(\mathcal{U})$

The question whether $h_{\mu}^{-}(\mathcal{U})=h_{\mu}^{+}(\mathcal{U})$ arose. In [HMRY] the authors continued the research on these concepts and proved, among other results, with aid of the Jewett-Krieger theorem, that if there exists a t.d.s, an invariant measure $\mu$ and an open cover $\mathcal{U}$ such that $h_{\mu}^{-}(\mathcal{U})<h_{\mu}^{+}(\mathcal{U})$ then one can find such a situation in a uniquely ergodic t.d.s. Recently, B.Weiss and E.Glasner [GW] showed that if $(X, T)$ is a t.d.s and $\mathcal{U}$ is any cover, then for any invariant measure $\mu h_{\mu}^{+}(\mathcal{U}) \leq h_{\text {top }}(\mathcal{U})$ and so combining these results one concludes that for a t.d.s and an open cover we have that $h_{\mu}^{-}(\mathcal{U})=h_{\mu}^{+}(\mathcal{U})$.

* Part of the author's MS.c thesis at the Hebrew University of Jerusalem. Email: ushapira@gmail.com. 
The measure theoretical entropy of a partition $\alpha$ in an ergodic m.t.d.s can be defined as: $\lim \frac{1}{n} \log \mathcal{N}\left(\alpha_{0}^{n-1}, \epsilon\right)$, where $0<\epsilon<1$ and $\mathcal{N}\left(\alpha_{0}^{n-1}, \epsilon\right)$ is the minimum number of atoms of $\alpha_{0}^{n-1}$ needed to cover $X$ up to a set of measure, less than $\epsilon$. (See [Ru]).

In this paper we follow this line and in section 4 define a notion of measure theoretical entropy for a cover $\mathcal{U}$ of an ergodic m.t.d.s as $h_{\mu}^{e}(\mathcal{U})=\lim \frac{1}{n} \log \mathcal{N}\left(\mathcal{U}_{0}^{n-1}, \epsilon\right)$ (where $0<\epsilon<1$ ). We prove (Theorem 4.2) the existence of the limit and its Independence of $\epsilon$, in a different way from $[\mathrm{Ru}]$ using Strong Rohlin Towers. This can serve as an alternative proof of the fact that the above definition of measure theoretical entropy of a partition in an ergodic m.t.d.s is well defined.

We show in a direct way that in the ergodic case the three notions: $h_{\mu}^{-}(\mathcal{U}), h_{\mu}^{+}(\mathcal{U}), h_{\mu}^{e}(\mathcal{U})$, coincide (Theorems 4.4, 4.5), and from the ergodic decomposition for $h_{\mu}^{-}(\mathcal{U}), h_{\mu}^{+}(\mathcal{U})$, proved in $[\mathrm{HMRY}]$, we deduce that $h_{\mu}^{-}(\mathcal{U})=h_{\mu}^{+}(\mathcal{U})$ in the general case (Corollary 5.2), and so, we can denote this number by $h_{\mu}(\mathcal{U}, T)$ or $h_{\mu}(\mathcal{U})$.

We also get an immediate proof of a slight generalization of the inequality $h_{\mu}(\mathcal{U}) \leq h_{\text {top }}(\mathcal{U})$, mentioned earlier, from $[\mathrm{GW}]$, to the non topological case (Theorem 6.1).

Acknowledgements : This paper was written as an M.Sc thesis at the Hebrew University of Jerusalem under the supervision of prof' Benjamin Weiss. I would like to thank prof' Weiss, for introducing me to the subject and for sharing with me his and Eli Glasner's valuable ideas.

\section{Preliminaries}

Recall that in the following a measure theoretical dynamical system, (m.t.d.s), is a four tuple $(X, \mathcal{B}, \mu, T)$, where $(X, \mathcal{B})$ is a standard space, $\mu$ is a probability measure on $(X, \mathcal{B})$ and $T$ is an invertible measure preserving transformation of $X$.

\subsection{Definition.}

- A cover of $X$ is a finite collection of measurable sets that cover $X$.

- The collection of covers of $X$ will be denoted by $\mathcal{C}_{X}$

- A partition of $X$ is a cover of $X$ whose elements are mutually disjoint.

- The collection of partitions of $X$ will be denoted by $\mathcal{P}_{X}$. Usually we denote covers by $\mathcal{U}, \mathcal{V}$ and partitions by $\alpha, \beta, \gamma$ etc.

- We say that a cover $\mathcal{U}$ is finer than $\mathcal{V}(\mathcal{U} \succeq \mathcal{V})$ if any element of $\mathcal{U}$ is contained in an element of $\mathcal{V}$.

- For any $\mathcal{U} \in \mathcal{C}_{X}$ and $k \in \mathbb{Z}$ we denote by $T^{k}(\mathcal{U})$ the cover whose elements are the sets of the form $T^{k}(U)$ where $U \in \mathcal{U}$.

- We define the join, $\mathcal{U} \vee \mathcal{V}$, of two covers $\mathcal{U}, \mathcal{V}$, to be the cover whose elements are sets of the form $U \cap V$ where $U \in \mathcal{U}$ and $V \in \mathcal{V}$.

- When the transformation $T$ is understood we denote, for $l>k$, the cover $T^{-k}(\mathcal{U}) \mathrm{V}$ $T^{-(k+1)}(\mathcal{U}) \cdots \vee T^{-l}(\mathcal{U})$, by $\mathcal{U}_{k}^{l}$. 
2.2. Definition. For $0<\delta<1$ define $H(\delta)=-\delta \log \delta-(1-\delta) \log (1-\delta)$. Note that $\lim _{\delta \rightarrow 0} H(\delta)=0$.

In the sequel, we will prove some combinatorial lemmas and often we will encounter the expression $\sum_{j \leq \delta K}\left(\begin{array}{c}K \\ j\end{array}\right)$. We shall make use of the next elementary lemma:

2.3. Lemma. (lemma 1.5.4 in [Sh1]): If $\delta<\frac{1}{2}$ then $\sum_{j \leq \delta K}\left(\begin{array}{c}K \\ j\end{array}\right) \leq 2^{H(\delta)}$.

2.4. Definition. A m.t.d.s $(X, \mathcal{B}, \mu, T)$ is said to be aperiodic, if for every $n \in \mathbb{N}$, $\mu\left(\left\{x \mid T^{n} x=x\right\}\right)=0$.

An ergodic system which is not aperiodic is easily seen to be a cyclic permutation on a finite number of atoms.

One of our main tools in practice, will be the Strong Rohlin Lemma ([Sh2] p.15):

2.5. Lemma. Let $(X, \mathcal{B}, \mu, T)$ be an ergodic, aperiodic system and let $\alpha \in \mathcal{P}_{X}$. Then for any $\delta>0$ and $n \in \mathbb{N}$, one can find a set $B \in \mathcal{B}$, such that $B, T B \ldots, T^{n-1} B$ are mutually disjoint, $\mu\left(\bigcup_{0}^{n-1} T^{i} B\right)>1-\delta$ and the distribution of $\alpha$ is the same as the distribution of the partition $\left.\alpha\right|_{B}$ that $\alpha$ induces on $B$.

The data $(n, \delta, B, \alpha)$ will be called, a strong Rohlin tower of height $n$ and error $\delta$ with respect to $\alpha$ and with $B$ as a base.

\section{Measure theoretical entropy of COVERS}

Let $(X, \mathcal{B}, \mu, T)$ be a m.t.d.s. The definitions and proofs in this section were introduced in $[R]$.

3.1. Definition. for $\mathcal{U} \in \mathcal{C}_{X}$ we define the entropy of $\mathcal{U}$ as:

$H_{\mu}(\mathcal{U})=i n f_{\alpha \succeq \mathcal{U}} H_{\mu}(\alpha)$.

\subsection{Proposition.}

(1) If $\mathcal{U}, \mathcal{V} \in \mathcal{C}_{X}$ then $H_{\mu}(\mathcal{U} \vee \mathcal{V}) \leq H_{\mu}(\mathcal{U})+H_{\mu}(\mathcal{V})$

(2) For every $\mathcal{U} \in \mathcal{C}_{X} H_{\mu}\left(T^{-1} \mathcal{U}\right)=H_{\mu}(\mathcal{U})$

3.3. Corollary. If $\mathcal{U} \in \mathcal{C}_{X}$ then the sequence $H_{\mu}\left(\mathcal{U}_{0}^{n-1}\right)$ is sub-additive.

3.4. Corollary. If $\mathcal{U} \in \mathcal{C}_{X}$ then the sequence $\frac{1}{n} H_{\mu}\left(\mathcal{U}_{0}^{n-1}\right)$ converges to in $f_{n} \frac{1}{n} H_{\mu}\left(\mathcal{U}_{0}^{n-1}\right)$.

Two ways of generalizing the definition of measure theoretical entropy of a partition to a cover are:

3.5. Definition. If $\mathcal{U} \in \mathcal{C}_{X}$, define

(1) $h_{\mu}^{-}(\mathcal{U}, T)=\lim \frac{1}{n} H_{\mu}\left(\mathcal{U}_{0}^{n-1}\right)$.

(2) $h_{\mu}^{+}(\mathcal{U}, T)=\inf _{\alpha \succeq \mathcal{U}} h_{\mu}(\alpha, T)$.

When $T$ is understood we usually omit it and write $h_{\mu}^{-}(\mathcal{U}), h_{\mu}^{+}(\mathcal{U})$.

We shall see later that in fact $h_{\mu}^{-}(\mathcal{U})=h_{\mu}^{+}(\mathcal{U})$. 


\subsection{Proposition.}

(1) $h_{\mu}^{-}(\mathcal{U}) \leq h_{\mu}^{+}(\mathcal{U})$.

(2) for any $m \in \mathbb{N} \quad h_{\mu}^{-}(\mathcal{U}, T)=\frac{1}{m} h_{\mu}^{-}\left(\mathcal{U}_{0}^{m-1}, T^{m}\right)$

(3) $h_{\mu}^{-}(\mathcal{U}, T)=\lim _{n} \frac{1}{n} h_{\mu}^{+}\left(\mathcal{U}_{0}^{n-1}, T^{n}\right)$

\section{THE ERGODIC CASE}

Throughout this section, $(X, \mathcal{B}, \mu, T)$, is an ergodic m.t.d.s.

For $\mathcal{U} \in \mathcal{C}_{X}$, we denote by $\mathcal{N}(\mathcal{U}, \epsilon, \mu)$, the minimum number of elements of $\mathcal{U}$, needed to cover all of $X$, up to a set of measure, less than $\epsilon$. When $\mu$ is understood we write $\mathcal{N}(\mathcal{U}, \epsilon)$.

By a strait forward calculation one deduces from [Sh1] p.51 the following:

4.1. Theorem. If $(X, \mathcal{B}, \mu, T)$ is an ergodic m.t.d.s and $\alpha \in \mathcal{P}_{X}$, then for any $0<\epsilon<1$, $h_{\mu}(\alpha, T)=\lim \frac{1}{n} \log \mathcal{N}\left(\alpha_{0}^{n-1}, \epsilon\right)$.

In view of this result, a natural way to generalize the definition of measure theoretical entropy of a partition to covers will be the following:

$$
h_{\mu}(\mathcal{U}, T)=\lim \frac{1}{n} \log \mathcal{N}\left(\mathcal{U}_{0}^{n-1}, \epsilon\right) .
$$

Where $0<\epsilon<1$. In order to do so we have to show that the above limit exists and is independent of $\epsilon$.

4.2. Theorem. For any $0<\epsilon<1$, the sequence $\frac{1}{n} \log \mathcal{N}\left(\mathcal{U}_{0}^{n-1}, \epsilon\right)$ converges and the limit is independent of $\epsilon$.

In order to prove this theorem we shall need a combinatorial lemma. Let us first introduce some terminology (in first reading the reader may skip the following discussion and turn to the discussion held after the proof of Lemma 4.3):

- We say that two intervals in $\mathbb{N}, I, J$ are separated if there is $n \in \mathbb{N}$ such that for any $i \in I, j \in J$ we have $i<n<j$ or $j<n<i$.

- We say that a collection $\left\{I_{i}\right\}_{i \in A}$ of intervals in $\mathbb{N}$ is a separated collection if any two of its elements are separated.

- We say that a collection $\left\{I_{i}\right\}_{i \in A}$ of subintervals of an interval $[1, K]$ is a $(\lambda, \epsilon)$ separated cover of $[1, K]$ (for $0<\lambda<1,0<\epsilon$ ), if it is separated and

$$
\left|\frac{\left|\cup I_{i}\right|}{K}-\lambda\right|<\epsilon
$$

- Given a vector $\vec{\lambda}=\left(\lambda_{1} \ldots \lambda_{l}\right)$, we denote

$$
\nu_{r}(\vec{\lambda})=\prod_{j=r}^{l}\left(1-\lambda_{j}\right)
$$


or just $\nu_{r}$ when $\vec{\lambda}$ is understood. For $r>l$ we set $\nu_{r}=1$. Note that for $j<l$ we have:

$$
\sum_{r=j+1}^{l} \lambda_{r} \nu_{r+1}=1-\nu_{j} .
$$

In the following combinatorial lemma, we will be given $l$ separated collections $\left\{I_{i}^{j}\right\}_{i \in A_{j}}$, $j=1 \ldots l$ of subintervals of a very long interval $[1, K]$. The knowledge about these collections is that the members of the $j$ 'th collection all have the same length, $N_{j}, N_{1}<<$ $N_{2} \cdots<<N_{l}$ and every collection is very "equally distributed" in $[1, K]$ in some sense.

We would like to extract, from these collections, a separated collection that will cover as much as we can, from $[1, K]$.

Let us denote by $\lambda_{j}$, the percentage of $[1, K]$, that is covered by the $j$ 'th collection and by $\vec{\lambda}$, the corresponding vector. Then, $\lambda_{l}=1-\nu_{l}$ percent of $[1, K]$ is covered by $\left\{I_{i}^{l}\right\}$. The complement is of size $K \nu_{l}$ and we could cover $\lambda_{l-1}$ percent of it with the $\left\{I_{i}^{l-1}\right\}$ 's. By now we covered $K\left(1-\nu_{l-1}\right)$ and we could cover $\lambda_{l-2}$ percent of the complement by the $\left\{I_{i}^{l-2}\right\}$ 's. So by now we covered $K\left(1-\nu_{l-2}\right)$ of $[1, K]$. We go on this way and extract a separated collection that covers $1-\nu_{1}$ percent of $[1, K]$. Let us now make these ideas precise.

4.3. Lemma. For any $l>0$, there exists a positive function $\varphi=\varphi\left(N_{1} \ldots N_{l}, \eta_{1} \ldots \eta_{l}, \epsilon\right)$ (where $N_{1}<N_{2} \cdots<N_{l} \in \mathbb{N}, \eta_{i}, \epsilon>0$ ) such that

$$
\limsup _{\epsilon \rightarrow 0} \limsup _{N_{1} \rightarrow \infty} \limsup _{\eta_{1} \rightarrow 0} \ldots \limsup _{N_{l} \rightarrow \infty} \limsup _{\eta_{l} \rightarrow 0} \varphi\left(N_{i}, \eta_{i}, \epsilon\right)=0 .
$$

and such that if $0<\lambda_{j}<1 j=1 \ldots l$ and $\left\{I_{i}^{j}\right\}_{i \in A_{j}}$ are separated collections of subintervals of $[1, K]$ that satisfy:

(a) For every $1 \leq j \leq l\left|I_{i}^{j}\right|=N_{j}$.

(b) For every $1 \leq j \leq l\left\{I_{i}^{j}\right\}$ is a $\left(\lambda_{j}, \epsilon\right)$-separated cover of $[1, K]$.

(c) For every $0 \leq j<r \leq l$, the number of subintervals, $J$, of $[1, K]$, of length $N_{r}$, which are not $\left(\lambda_{j}, \epsilon\right)$-separately covered by $\left\{I_{i}^{j} \subset J\right\}$ is less than $\eta_{r} K$.

then there are sets $\tilde{A}_{j} \subset A_{j} j=1 \ldots l$, such that $\left\{\left\{I_{i}^{j}\right\}_{i \in \tilde{A}_{j}}\right\}_{j=1}^{l}$ is a separated collection and $[1, K]$ is $\left(\left(1-\nu_{1}(\vec{\lambda})\right), \varphi\left(N_{i}, \eta_{i}, \epsilon\right)\right)$-separately covered by $\left\{\left\{I_{i}^{j}\right\}_{i \in \tilde{A}_{j}}\right\}_{j=1}^{l}$.

Proof. We will build the $\tilde{A}_{j}$ 's by recursion, starting with $j=l$. Define $\tilde{A}_{l}=A_{l}$. Then from (b) we have that $\left|\frac{N_{l}\left|\tilde{A}_{l}\right|}{K}-\lambda_{l}\right|<\epsilon$. So if we will define $f_{l}\left(N_{i}, \eta_{i}, \epsilon\right)=\epsilon$, then $f_{l}$ satisfies $(*)$ and $[1, K]$ is $\left(\lambda_{l} \nu_{l+1}, f_{l}\left(N_{i}, \eta_{i}, \epsilon\right)\right)$-separately covered by $\left\{I_{i}^{l}\right\}_{i \in \tilde{A}_{l}}$. Now, suppose we have defined $\tilde{A}_{l} \ldots \tilde{A}_{j+1}$ and positive functions $f_{l} \ldots f_{j+1}$, that satisfy $(*)$, such that $\left\{\left\{I_{i}^{r}\right\}_{i \in \tilde{A}_{r}}\right\}_{r=j+1}^{l}$, is a separated collection and for every $j+1 \leq r \leq l,[1, K]$ is $\left(\lambda_{r} \nu_{r+1}, f_{r}\left(N_{i}, \eta_{i}, \epsilon\right)\right)$-separately covered by $\left\{I_{i}^{r}\right\}_{i \in \tilde{A}_{r}}$. Define now,

$$
\tilde{A}_{j}=\left\{i \in A_{j} \mid I_{i}^{j} \text { is separated from }\left\{I_{s}^{r}\right\}_{s \in \tilde{A}_{r}}, r=j+1 \ldots l\right\} .
$$


We want to estimate the size of $\tilde{A}_{j}$.

Estimation from below: Choose $j+1 \leq r \leq l$ and divide the members of $\left\{I_{i}^{r}\right\}_{i \in \tilde{A}_{r}}$ to good ones and bad ones according to (c), i.e, $I_{s}^{r}$ is good if it is $\left(\lambda_{j}, \epsilon\right)$-separately covered by $\left\{I_{i}^{j} \subset I_{s}^{r}\right\}$. We have at most $\eta_{r} K, I_{i}^{r}$ 's, which are bad and at most $\left|\tilde{A}_{r}\right|, I_{i}^{r}$ 's, which are good. Every bad $I_{i}^{r}$ rules out at most $\frac{N_{r}}{N_{j}}+2 i$ 's in $A_{j}$ from being in $\tilde{A}_{j}$. Every good $I_{i}^{r}$ rules out at most $\frac{N_{r}}{N_{j}}\left(\lambda_{j}+\epsilon\right)+2, i$ 's in $A_{j}$ from being in $\tilde{A}_{j}$. In total, the maximum number of $i$ 's in $A_{j}$ that are not in $\tilde{A}_{j}$ is at most:

$$
\sum_{r=j+1}^{l}\left|\tilde{A}_{r}\right|\left(\frac{N_{r}}{N_{j}}\left(\lambda_{j}+\epsilon\right)+2\right)+\eta_{r} K\left(\frac{N_{r}}{N_{j}}+2\right)=(* *)
$$

Note that because $[1, K]$ is $\left(\lambda_{r} \nu_{r+1}, f_{r}\right)$-separately covered by $\left\{I_{i}^{r}\right\}_{i \in \tilde{A}_{r}}$, we must have

$$
\left|\tilde{A}_{r}\right| \leq \frac{K}{N_{r}}\left(\lambda_{r} \nu_{r+1}+f_{r}\right) .
$$

Using this we get:

$$
\begin{gathered}
(* *) \leq \sum_{r=j+1}^{l} \frac{K}{N_{r}}\left(\lambda_{r} \nu_{r+1}+f_{r}\right)\left(\frac{N_{r}}{N_{j}}\left(\lambda_{j}+\epsilon\right)+2\right)+\eta_{r} K\left(\frac{N_{r}}{N_{j}}+2\right) \\
=\sum_{r=j+1}^{l} \frac{K}{N_{j}} \lambda_{r} \nu_{r+1}\left(\lambda_{j}+\epsilon\right)+\frac{K}{N_{j}}\left(\lambda_{j}+\epsilon\right) f_{r}+\frac{2 K}{N_{r}}\left(\lambda_{r} \nu_{r+1}+f_{r}\right)+\frac{K}{N_{j}} \eta_{r} N_{r}+2 \eta_{r} K \\
=\frac{K}{N_{j}} \lambda_{j}\left(\sum_{r=j+1}^{l} \lambda_{r} \nu_{r+1}\right) \\
+\frac{K}{N_{j}} \sum_{r=j+1}^{l}\left\{\epsilon \lambda_{r} \nu_{r+1}+\left(\lambda_{j}+\epsilon\right) f_{r}+2 \frac{N_{j}}{N_{r}}\left(\lambda_{r} \nu_{r+1}+f_{r}\right)+\eta_{r}\left(N_{r}+2 N_{j}\right)\right\}=(\aleph)
\end{gathered}
$$

as mentioned earlier $\sum_{j+1}^{l} \lambda_{r} \nu_{r+1}=1-\nu_{j}$ so we have that:

$$
\begin{gathered}
\left|\tilde{A}_{j}\right| \geq\left|A_{j}\right|-(\aleph) \geq \frac{K}{N_{j}}\left(\lambda_{j}-\epsilon\right)-(\aleph) \\
=\frac{K}{N_{j}}\left\{\lambda_{j} \nu_{j}-\left\{\epsilon+\sum_{r=j+1}^{l}\left\{\epsilon \lambda_{r} \nu_{r+1}+\left(\lambda_{j}+\epsilon\right) f_{r}+2 \frac{N_{j}}{N_{r}}\left(\lambda_{r} \nu_{r+1}+f_{r}\right)+\eta_{r}\left(N_{r}+2 N_{j}\right)\right\}\right\}\right\}
\end{gathered}
$$

note that

$$
\begin{gathered}
\mid\left(\epsilon+\sum_{r=j+1}^{l}\left\{\epsilon \lambda_{r} \nu_{r+1}+\left(\lambda_{j}+\epsilon\right) f_{r}+2 \frac{N_{j}}{N_{r}}\left(\lambda_{r} \nu_{r+1}+f_{r}\right)+\eta_{r}\left(N_{r}+2 N_{j}\right)\right\} \mid\right. \\
\leq \epsilon+\sum_{r=j+1}^{l}\left\{\epsilon+(1+\epsilon) f_{r}+2 \frac{N_{j}}{N_{r}}\left(1+f_{r}\right)+\eta_{r}\left(N_{r}+2 N_{j}\right)\right\}
\end{gathered}
$$


so if we will denote the last expression by $\tilde{f}_{j}\left(N_{i}, \eta_{i}, \epsilon\right)$, then we see that $\tilde{f}_{j}$ satisfies $(*)$ and $\left|\tilde{A}_{j}\right| \geq \frac{K}{N_{j}}\left(\lambda_{j} \nu_{j+1}-\tilde{f}_{j}\right)$.

Estimation from above: For every $j+1 \leq r \leq l$, we have that $\left|\tilde{A}_{r}\right| \geq \frac{K}{N_{r}}\left(\lambda_{r} \nu_{r+1}-f_{r}\right)$ and the number of bad $I_{i}^{r}$ 's is at most $\eta_{r} K$, so we must have at least $\frac{K}{N_{r}}\left(\lambda_{r} \nu_{r+1}-f_{r}\right)-\eta_{r} K$ good $I_{i}^{r}$ 's. Every good $I_{i}^{r}$, rules out at least $\frac{N_{r}}{N_{j}}\left(\lambda_{j}-\epsilon\right) i$ 's in $A_{j}$ from being in $\tilde{A}_{j}$. So the number of $i$ 's in $A_{j}$ that are not in $\tilde{A}_{j}$ is at least:

$$
\sum_{r=j+1}^{l} \frac{N_{r}}{N_{j}}\left(\lambda_{j}-\epsilon\right)\left\{\frac{K}{N_{r}}\left(\lambda_{r} \nu_{r+1}-f_{r}\right)-\eta_{r} K\right\}
$$

and so

$$
\begin{gathered}
\left|\tilde{A}_{j}\right| \leq\left|A_{j}\right|-\sum_{r=j+1}^{l} \frac{N_{r}}{N_{j}}\left(\lambda_{j}-\epsilon\right)\left\{\frac{K}{N_{r}}\left(\lambda_{r} \nu_{r+1}-f_{r}\right)-\eta_{r} K\right\} \\
\leq \frac{K}{N_{j}}\left(\lambda_{j}+\epsilon\right)-\sum_{r=j+1}^{l}\left\{\frac{K}{N_{j}}\left(\lambda_{j}\left(\lambda_{r} \nu_{r+1}-f_{r}\right)-\epsilon\left(\lambda_{r} \nu_{r+1}-f_{r}\right)\right)-\frac{K}{N_{j}} \eta_{r} N_{r}\left(\lambda_{j}-\epsilon\right)\right\} \\
=\frac{K}{N_{j}}\left\{\lambda_{j}\left(1-\sum_{r=j+1}^{l} \lambda_{r} \nu_{r+1}\right)+\epsilon+\sum_{r=j+1}^{l}\left(\lambda_{j} f_{r}+\epsilon\left(\lambda_{r} \nu_{r+1}-f_{r}\right)+\eta_{r} N_{r}\left(\lambda_{j}-\epsilon\right)\right)\right\} \\
\leq \frac{K}{N_{j}}\left\{\lambda_{j} \nu_{j+1}+\epsilon+\sum_{r=j+1}^{l}\left(f_{r}+\epsilon\left(1+f_{r}\right)+\eta_{r} N_{r}(1+\epsilon)\right)\right\}
\end{gathered}
$$

so if we will denote

$$
\left.\hat{f}_{j}\left(N_{i}, \eta_{i}, \epsilon\right)=\epsilon+\sum_{r=j+1}^{l}\left(f_{r}+\epsilon\left(1+f_{r}\right)+\eta_{r} N_{r}(1+\epsilon)\right)\right\}
$$

then $\hat{f}_{j}$ satisfies $(*)$ and $\left|\tilde{A}_{j}\right| \leq \frac{K}{N_{j}}\left(\lambda_{j} \nu_{j+1}+\hat{f}_{j}\right)$. Define $f_{j}=\max \left(\tilde{f}_{j}, \hat{f}_{j}\right)$ and then we have that $f_{j}$ satisfies $(*)$ and

$$
\left|\frac{\left|\tilde{A}_{j}\right| N_{j}}{K}-\lambda_{j} \nu_{j+1}\right| \leq f_{j}
$$

We have defined $\tilde{A}_{j} \subset A_{j}$ and a positive function $f_{j}$, that satisfies $(*)$, such that $\left\{\left\{I_{i}^{r}\right\}_{i \in \tilde{A}_{r}}\right\}_{r=j}^{l}$ is a separated collection and $[1, K]$ is $\left(\lambda_{j} \nu_{j+1}, f_{j}\right)$-separately covered by $\left\{I_{i}^{j}\right\}_{i \in \tilde{A}_{j}}$. We continue this way and define sets $\tilde{A}_{j} \subset A_{j}$ and positive functions $f_{j}, j=1 \ldots l$, such that $\left\{\left\{I_{i}^{j}\right\}_{i \in \tilde{A}_{j}}\right\}_{j=1}^{l}$, is a separated collection and $[1, K]$ is $\left(\lambda_{j} \nu_{j+1}, f_{j}\right)$-separately covered by $\left\{I_{i}^{j}\right\}_{i \in \tilde{A}_{j}}$.

Note that this means:

$$
K\left(\sum_{j=1}^{l} \lambda_{j} \nu_{j+1}-\sum_{j=1}^{l} f_{r}\right) \leq\left|\bigcup_{j=1}^{l} \bigcup_{i \in \tilde{A}_{j}} I_{i}^{j}\right| \leq K\left(\sum_{j=1}^{l} \lambda_{j} \nu_{j+1}+\sum_{j=1}^{l} f_{r}\right)
$$


and so, if we will define $\varphi=\sum f_{j}$, then $\varphi$ satisfies $(*)$ and $\left\{\left\{I_{i}^{j}\right\}_{i \in \tilde{A}_{j}}\right\}_{j=1}^{l}$, is a $\left(1-\nu_{1}, \varphi\right)$ separated cover of $[1, K]$.

Before turning to the proof of theorem 4.2, let us present some terminology. In the following $\mathcal{U}=\left\{U_{1} \ldots U_{M}\right\}$, is a cover of $X$. For any $\rho>0$, we can find a partition $\beta \succeq \mathcal{U}$, such that $\mathcal{N}(\mathcal{U}, \rho)=\mathcal{N}(\beta, \rho)$. Namely, we choose a subset of $\mathcal{U}$, of $N=\mathcal{N}(\mathcal{U}, \rho)$ elements, that covers $X$ up to a set of measure $<\rho,\left\{U_{i 1} \ldots U_{i N}\right\}$ and define $C_{1}=U_{i 1}$, $C_{j}=U_{i j} \backslash \bigcup_{m=1}^{j-1} U_{i m}, j=2 \ldots N$. The $C_{j}$ 's are disjoint, $C_{j} \subset U_{i j}$ and $\bigcup_{1}^{N} C_{j}=\bigcup_{j=1}^{N} U_{i j}$. Extend the collection $\left\{C_{j}\right\}_{j=1}^{N}$ to a partition, $\beta$, refining $\mathcal{U}$, in some way. Then, because $\beta \succeq \mathcal{U}$, we have $\mathcal{N}(\beta, \rho) \geq N$ and from our construction, it follows that $\mathcal{N}(\beta, \rho) \leq N$.

- We call such a partition, a $\rho$-good partition for $\mathcal{U}$.

If $(X, \mathcal{B}, \mu, T)$ is aperiodic and $N \in \mathbb{N}, \rho, \delta>0$ are given, then for a $\rho$-good partition $\beta$, for $\mathcal{U}_{0}^{N-1}$, we can construct a strong Rohlin tower with height $N+1$ and error $<\delta$. Let $\tilde{B}$ denote the base of the tower and let $B \subset \tilde{B}$ be a union of $\mathcal{N}(\beta, \rho)$ atoms of $\left.\beta\right|_{\tilde{B}}$ that covers $\tilde{B}$ up to a set of measure, less than $\rho \mu(\tilde{B})$.

- We call $(\beta, \tilde{B}, B)$, a good base for $(\mathcal{U}, N, \rho, \delta)$.

- For a set $J \subset \mathbb{N}$, a $(\mathcal{U}, J)$-name, is a function $f: J \rightarrow\{1 \ldots M\}$.

- $f$ is a name of $x \in X$, if $x \in \bigcap_{j \in J} T^{-j} U_{f(j)}$.

- We denote the set of elements of $X$ with $f$ as a name by $S_{f}$.

- A set of $(\mathcal{U}, J)$-names, $\left\{f_{i}\right\}$, covers a set $C \in \mathcal{B}$, if $C \subset \bigcup_{i} S_{f_{i}}$.

In the sequel, we will want to estimate the number of elements of $\mathcal{U}_{0}^{N-1}$, needed to cover a set $C \in \mathcal{B}$, i.e, we will want to estimate the number of $(\mathcal{U},[0, N-1])$-names needed to cover $C$. The usual way to do so is to find a collection of disjoint sets $J_{i} \subset[0, N-1]$ $i=1 \ldots m$, that covers most of $[0, N-1]$, such that we can bound the number of $\left(\mathcal{U}, J_{i}\right)$ names needed to cover $C$. If we can cover $C$ by $R_{i},\left(\mathcal{U}, J_{i}\right)$-names, $\left\{f_{m}^{i}\right\}_{m=1}^{R_{i}}$, then the set $\Gamma=\left\{f:[0, N-1] \rightarrow\{1 \ldots M\}|f|_{J_{i}} \in\left\{f_{m}^{i}\right\}_{m=1}^{R_{i}}\right\}$, of $(\mathcal{U},[0, N-1])$-names, covers $C$ and contains $\prod R_{i} \cdot M^{N-\sum\left|J_{i}\right|}$ elements.

This situation occurs in our proofs in the following way: Let $(\beta, \tilde{B}, B)$, be a good base for $(\mathcal{U}, N, \rho, \delta)$ and $K>>N$. Set $C$ to be the set of elements of $X$ that visits $B$ at times $i_{1}<\cdots<i_{m}$ between 0 to $K-N$ (under the action of $T$ ). Then we can cover $C$ by no more than $\mathcal{N}(\beta, \rho),\left(\mathcal{U},\left[i_{j}, i_{j}+N-1\right]\right)$-names. We can now turn to the proof of theorem 4.2 .

Proof. (theorem 4.2): If $(X, \mathcal{B}, \mu, T)$ is periodic, it follows from the ergodicity, that the system is a cyclic permutation on a finite set of atoms and for every $0<\epsilon<1$ we have $\lim \frac{1}{n} \log \mathcal{N}\left(\mathcal{U}_{0}^{n-1}, \epsilon\right)=0$. We assume, then, that the system is aperiodic and thus we are able to use the Strong Rohlin Lemma. Given $0<\rho_{2}<\rho_{1}<1$, we need to show that the limits: $\lim \frac{1}{n} \log \mathcal{N}\left(\mathcal{U}_{0}^{n-1}, \rho_{i}\right) i=1,2$, exist and are equal. Note that for every $n$, we have that $\mathcal{N}\left(\mathcal{U}_{0}^{n-1}, \rho_{1}\right) \leq \mathcal{N}\left(\mathcal{U}_{0}^{n-1}, \rho_{2}\right)$ and thus $\limsup \frac{1}{n} \log \mathcal{N}\left(\mathcal{U}_{0}^{n-1}, \rho_{1}\right) \leq$ $\liminf \frac{1}{n} \log \mathcal{N}\left(\mathcal{U}_{0}^{n-1}, \rho_{2}\right)$, so it's enough to prove that

$$
\limsup \frac{1}{n} \log \mathcal{N}\left(\mathcal{U}_{0}^{n-1}, \rho_{2}\right) \leq \liminf \frac{1}{n} \log \mathcal{N}\left(\mathcal{U}_{0}^{n-1}, \rho_{1}\right) .
$$


Let $0<\epsilon_{0}<\frac{1}{2}$, be given and denote:

$h_{0}=\liminf \frac{1}{n} \log \mathcal{N}\left(\mathcal{U}_{0}^{n-1}, \rho_{1}\right), L=\left\{n \in \mathbb{N}|| h_{0}-\frac{1}{n} \log \mathcal{N}\left(\mathcal{U}_{0}^{n-1}, \rho_{1}\right) \mid<\epsilon_{0}\right\}$,

so $L$ contains arbitrarily large numbers. Choose $\ell \in \mathbb{N}$, large enough so that

$$
\left(\frac{1}{2}\left(1+\rho_{1}\right)\right)^{\ell} \log M<\epsilon_{0}, \quad\left(\frac{1}{2}\left(1+\rho_{1}\right)\right)^{\ell}+\epsilon_{0}<\frac{1}{2} \quad(*) .
$$

The towers construction: Remember the function $\varphi$ from the combinatorial lemma (Lemma 4.3). It satisfies:

$$
\limsup _{\epsilon \rightarrow 0} \limsup _{N_{1} \rightarrow \infty} \limsup _{\eta_{1} \rightarrow 0} \ldots \limsup _{N_{\ell} \rightarrow \infty} \limsup _{\eta_{\ell} \rightarrow 0} \varphi\left(N_{i}, \eta_{i}, \epsilon\right)=0
$$

so we can choose $\epsilon>0$, small enough, such that

$$
\limsup _{N_{1} \rightarrow \infty} \limsup _{\eta_{1} \rightarrow 0} \ldots \limsup _{N_{\ell} \rightarrow \infty} \limsup _{\eta_{\ell} \rightarrow 0} \varphi\left(N_{i}, \eta_{i}, \epsilon\right)<\epsilon_{0} .
$$

Choose a small enough $\delta>0$ (in a manner specified later). Choose $N_{1} \in L$, large enough, such that

$$
\limsup _{\eta_{1} \rightarrow 0} \ldots \limsup _{N_{\ell} \rightarrow \infty} \limsup _{\eta_{\ell} \rightarrow 0} \varphi\left(N_{i}, \eta_{i}, \epsilon\right)<\epsilon_{0} .
$$

Find a good base $\left(\beta_{1}, \tilde{B}_{1}, B_{1}\right)$, for $\left(\mathcal{U}, N_{1}, \rho_{1}, \delta\right)$. Choose $\eta_{1}>0$, small enough, such that

$$
\limsup _{N_{2} \rightarrow \infty} \limsup _{\eta_{2} \rightarrow 0} \ldots \limsup _{N_{\ell} \rightarrow \infty} \limsup _{\eta_{\ell} \rightarrow 0} \varphi\left(N_{i}, \eta_{i}, \epsilon\right)<\epsilon_{0} .
$$

From the ergodicity, we can choose $N_{2} \in L$, large enough, such that

- $\lim \sup _{\eta_{2} \rightarrow 0} \ldots \lim \sup _{N_{\ell} \rightarrow \infty} \lim \sup _{\eta_{\ell} \rightarrow 0} \varphi\left(N_{i}, \eta_{i}, \epsilon\right)<\epsilon_{0}$.

- $\mu\left\{x|| \frac{1}{N_{2}} \sum_{r=0}^{N_{2}-N_{1}} \chi_{B_{1}}\left(T^{r} x\right)-\mu\left(B_{1}\right) \mid<\frac{\epsilon}{N_{1}}\right\}>1-\eta_{1}$.

Find a good base, $\left(\beta_{2}, \tilde{B}_{2}, B_{2}\right)$, for $\left(\mathcal{U}, N_{2}, \rho_{1}, \delta\right)$. Choose $\eta_{2}>0$, small enough, such that

$$
\limsup _{N_{3} \rightarrow \infty} \limsup _{\eta_{3} \rightarrow 0} \ldots \limsup _{N_{\ell} \rightarrow \infty} \limsup _{\eta_{\ell} \rightarrow 0} \varphi\left(N_{i}, \eta_{i}, \epsilon\right)<\epsilon_{0} .
$$

Again, from the ergodicity, we can choose $N_{3} \in L$, such that

- $\lim \sup _{\eta_{3} \rightarrow 0} \ldots \lim \sup _{N_{\ell} \rightarrow \infty} \lim \sup _{\eta_{\ell} \rightarrow 0} \varphi\left(N_{i}, \eta_{i}, \epsilon\right)<\epsilon_{0}$.

- $\mu\left\{x|| \frac{1}{N_{3}} \sum_{r=0}^{N_{3}-N_{j}} \chi_{B_{j}}\left(T^{r} x\right)-\mu\left(B_{j}\right) \mid<\frac{\epsilon}{N_{j}} j=1,2\right\}>1-\eta_{2}$.

In this way we construct, inductively, $N_{1}<N_{2} \cdots<N_{\ell}$ (all from $L$ ), $\eta_{1} \ldots \eta_{\ell}$ and good bases $\left(\beta_{j}, \tilde{B}_{j}, B_{j}\right)$, for $\left(\mathcal{U}, N_{j}, \rho_{1}, \delta\right)$, such that $\varphi\left(N_{i}, \eta_{i}, \epsilon\right)<\epsilon_{0}$ and if we denote

$$
F_{j}=\left\{x|| \frac{1}{N_{j}} \sum_{r=0}^{N_{j}-N_{i}} \chi_{B_{i}}\left(T^{r} x\right)-\mu\left(B_{i}\right) \mid<\frac{\epsilon}{N_{i}} i=1 \ldots j-1\right\}
$$

then, $\mu\left(F_{j}\right)>1-\eta_{j}$.

Define

$$
E_{K}=\left\{x\left|\frac{1}{K} \sum_{r=0}^{K-N_{j}} \chi_{F_{j}}\left(T^{r} x\right)>1-\eta_{j},\right| \frac{1}{K} \sum_{r=0}^{K-N_{j}} \chi_{B_{j}}\left(T^{r} x\right)-\mu\left(B_{j}\right) \mid<\frac{\epsilon}{N_{j}} \quad j=1 \ldots \ell\right\} .
$$


From the ergodicity, we know that there is a $K_{0}$, such that, for any $K>K_{0}$, we have $\mu\left(E_{K}\right)>\rho_{2}$. Fix $K>K_{0}$, we shall show that we can cover $E_{K}$, by "few" $(\mathcal{U},[0, K-1])$ names. For a fixed $x \in E_{K}$ denote

$$
A_{j}=\left\{0 \leq m \leq K-N_{j} \mid T^{m} x \in B_{j}\right\}
$$

and for every $i \in A_{j}$, let $I_{i}^{j}=\left[i, i+N_{j}-1\right]$. We claim that the collections $\left\{I_{i}^{j}\right\}_{i \in A_{j}}$ $j=1 \ldots \ell$, satisfies conditions $(a),(b),(c)$ from the combinatorial lemma (lemma 4.3), with $\lambda_{j}=N_{j} \mu\left(B_{j}\right)$. To see this, note first, that because the height of the $j$ 'th tower was $N_{j}+1$, we have that each collection $\left\{I_{i}^{j}\right\}_{i \in A_{j}}$, is separated.

(a) By definition $\left|I_{i}^{j}\right|=N_{j}$.

(b) because $x \in E_{k}$, we know that $\left|\frac{1}{K} \sum_{r=0}^{K-N_{j}} \chi_{B_{j}}\left(T^{r} x\right)-\mu\left(B_{j}\right)\right|<\frac{\epsilon}{N_{j}}$ and thus, $\mid \frac{N_{j}\left|A_{j}\right|}{K}-$ $\lambda_{j} \mid<\epsilon$. So the $\left\{I_{i}^{j}\right\}_{i \in A_{j}}$ forms a $\left(\lambda_{j}, \epsilon\right)$-separated cover of $[0, K-1]$.

(c) For $1<r \leq \ell$, we know from the fact that $x \in E_{K}$, that $\frac{1}{K} \sum_{s=0}^{K-N_{r}} \chi_{F_{r}}\left(T^{s} x\right)>1-\eta_{r}$ and thus we have $\frac{1}{K} \sum_{s=0}^{K-N_{r}} \chi_{F_{r}^{c}}\left(T^{s} x\right)<\eta_{r}$. If we use the definition of $F_{r}$, this becomes

$$
\frac{1}{K} \#\left\{0 \leq s \leq K-N_{r}|\exists 1 \leq j \leq r-1| \frac{1}{N_{r}} \sum_{i=0}^{N_{r}-N_{j}} \chi_{B_{j}}\left(T^{i+s} x\right)-\mu\left(B_{j}\right) \mid \geq \frac{\epsilon}{N_{j}}\right\}<\eta_{r}
$$

or equivalently

$$
\#\left\{0 \leq s \leq K-N_{r}|\exists 1 \leq j \leq r-1| \frac{N_{j}}{N_{r}} \#\left\{i \mid i+s \in A_{j}\right\}-\lambda_{j} \mid \geq \epsilon\right\}<\eta_{r} K
$$

so if we choose $1 \leq j<r \leq \ell$, we must have

$$
\#\left\{J \subset[0, K-1]|| J\left|=N_{r},\right| \frac{N_{j}}{N_{r}} \#\left\{i \mid I_{i}^{j} \subset J\right\}-\lambda_{j} \mid \geq \epsilon\right\}<\eta_{r} K .
$$

In words, the number of subintervals of $[0, K-1]$ of length $N_{r}, J$, which are not $\left(\lambda_{j}, \epsilon\right)$ separately covered, by those $I_{i}^{j}$ which are contained in $J$ is less than $\eta_{r} K$, as we wanted. Using the combinatorial lemma, we can choose for every $x \in E_{K}$ a separated collection $\left\{\left\{I_{i}^{j}(x)\right\}_{i \in \tilde{A}_{j}}\right\}_{j=1}^{\ell}$ that covers at least $K\left(1-\nu_{1}(\vec{\lambda})-\epsilon_{0}\right)$ elements of $[0, K-1]$. Because these collections are separated, there is a $1-1$ correspondence between them and their complements. Hence, the number of such covers is less than

$$
\psi\left(K, \lambda_{j}, \epsilon_{0}\right)=\sum_{j \leq\left(\nu_{1}+\epsilon_{0}\right) K}\left(\begin{array}{c}
K \\
j
\end{array}\right)
$$

Fix such a collection $\left\{\left\{I_{i}^{j}\right\}_{i \in \tilde{A}_{j}}\right\}_{j=1}^{\ell}$ and set

$$
C=\left\{x \in E_{K} \mid\left\{I_{i}^{j}(x)\right\}=\left\{I_{i}^{j}\right\}\right\} .
$$

From the construction we see that for every $1 \leq j \leq \ell$ we can cover $B_{j}$ by no more than $2^{N_{j}\left(h_{0}+\epsilon_{0}\right)}\left(\mathcal{U},\left[0, N_{j}-1\right]\right)$-names, thus we can cover $C$ by no more than $2^{N_{j}\left(h_{0}+\epsilon_{0}\right)}$ 
$\left(\mathcal{U}, I_{i}^{j}\right)$-names. So the number of $(\mathcal{U},[0, K-1])$-names, needed to cover $C$ is at most

$$
\begin{gathered}
\prod_{j=1}^{\ell}\left(2^{N_{j}\left(h_{0}+\epsilon_{0}\right)}\right)^{\left|\tilde{A}_{j}\right|} \cdot M^{K\left(\nu_{1}+\epsilon_{0}\right)}=2^{\left(\sum_{j} N_{j}\left|\tilde{A}_{j}\right|\right)\left(h_{0}+\epsilon_{0}\right)} \cdot M^{K\left(\nu_{1}+\epsilon_{0}\right)} \\
\leq 2^{K\left(h_{0}+\epsilon_{0}\right)} \cdot M^{K\left(\nu_{1}+\epsilon_{0}\right)} .
\end{gathered}
$$

Finally we get from this and $(* *)$ that

$$
\mathcal{N}\left(\mathcal{U}_{0}^{K-1}, \rho_{2}\right) \leq \psi\left(K, \lambda_{j}, \epsilon_{0}\right) \cdot 2^{K\left(h_{0}+\epsilon_{0}\right)} \cdot M^{K\left(\nu_{1}+\epsilon_{0}\right)}
$$

and so

$$
\frac{1}{K} \log \mathcal{N}\left(\mathcal{U}_{0}^{K-1}, \rho_{2}\right) \leq \frac{1}{K} \log \psi\left(K, \lambda_{j}, \epsilon_{0}\right)+h_{0}+\epsilon_{0}+\nu_{1} \log M+\epsilon_{0} \log M
$$

If, in the construction of the towers, we choose $\delta$ small enough and $N_{1}$ large enough, we can ensure that $\lambda_{j}=N_{j} \mu\left(B_{j}\right)>\frac{1-\rho_{1}}{2}$ and thus $1-\lambda_{j}<\frac{1+\rho_{1}}{2} \Rightarrow \nu_{1}<\left(\frac{1+\rho_{1}}{2}\right)^{\ell}$ and so, from $(*)$ we have that

$$
\nu_{1} \log M<\epsilon_{0} \quad \nu_{1}+\epsilon_{0} \leq \frac{1}{2}
$$

hence, from lemma 2.3

$$
\psi\left(K, \lambda_{j}, \epsilon_{0}\right) \leq 2^{K \cdot H\left(\left(\frac{1+\rho_{1}}{2}\right)^{\ell}+\epsilon_{0}\right)}
$$

hence

$$
\begin{gathered}
\frac{1}{K} \log \mathcal{N}\left(\mathcal{U}_{0}^{K-1}, \rho_{2}\right) \leq h_{0}+\epsilon_{0}(2+\log M)+H\left(\left(\frac{1+\rho_{1}}{2}\right)^{\ell}+\epsilon_{0}\right) \Rightarrow \\
\limsup \frac{1}{K} \log \mathcal{N}\left(\mathcal{U}_{0}^{K-1}, \rho_{2}\right) \leq h_{0}+\epsilon_{0}(2+\log M)+H\left(\left(\frac{1+\rho_{1}}{2}\right)^{\ell}+\epsilon_{0}\right)
\end{gathered}
$$

letting $\ell \rightarrow \infty$ and $\epsilon_{0} \rightarrow 0$ we get

$$
\limsup _{K} \frac{1}{K} \log \mathcal{N}\left(\mathcal{U}_{0}^{K-1}, \rho_{2}\right) \leq h_{0}
$$

as desired.

After proving theorem 4.2, we can define, for an ergodic m.t.d.s, $(X, \mathcal{B}, \mu, T)$ and a cover $\mathcal{U}=\left\{U_{1} \ldots U_{M}\right\}$ of $X$, a notion of measure theoretical entropy in the following way:

$$
h_{\mu}^{e}(\mathcal{U}, T)=\lim \frac{1}{n} \log \mathcal{N}\left(\mathcal{U}_{0}^{n-1}, \epsilon\right) \quad \text { where } \quad 0<\epsilon<1 .
$$

Often we omit $T$ and write $h_{\mu}^{e}(\mathcal{U})$.

4.4. Theorem. $h_{\mu}^{e}(\mathcal{U})=h_{\mu}^{+}(\mathcal{U})$ 
Proof. As before, if the system is periodic then $h_{\mu}^{e}(\mathcal{U})=h_{\mu}^{+}(\mathcal{U})=0$. We assume, then ,that the system is aperiodic. For every partition $\alpha \succeq \mathcal{U}, n \in \mathbb{N}$ and $0<\epsilon<1$, we have that $\mathcal{N}\left(\mathcal{U}_{0}^{n-1}, \epsilon\right) \leq \mathcal{N}\left(\alpha_{0}^{n-1}, \epsilon\right)$ and therefore

$$
\begin{gathered}
h_{\mu}^{e}(\mathcal{U})=\lim \frac{1}{n} \log \mathcal{N}\left(\mathcal{U}_{0}^{n-1}, \epsilon\right) \leq \lim \frac{1}{n} \log \mathcal{N}\left(\alpha_{0}^{n-1}, \epsilon\right)=h_{\mu}(\alpha) \\
\Rightarrow h_{\mu}^{e}(\mathcal{U}) \leq h_{\mu}^{+}(\mathcal{U})
\end{gathered}
$$

To prove the other inequality, we shall show that for a given $0<\epsilon<\frac{1}{4}$ and $n \in \mathbb{N}$ we have:

$$
h_{\mu}^{+}(\mathcal{U}) \leq \frac{1}{n} \log \mathcal{N}\left(\mathcal{U}_{0}^{n-1}, \epsilon\right)+\sqrt{\epsilon} \cdot \log M+H(\sqrt{\epsilon}) .
$$

Once we prove $(*)$, we are done, for letting $n \rightarrow \infty$ we get $h_{\mu}^{+}(\mathcal{U}) \leq h_{\mu}^{e}(\mathcal{U})+\sqrt{\epsilon} \cdot \log M+$ $H(\sqrt{\epsilon})$ and now, letting $\epsilon \rightarrow 0$ we get $h_{\mu}^{+}(\mathcal{U}) \leq h_{\mu}^{e}(\mathcal{U})$ as desired.

Proof of $(*)$ : choose $\delta>0$, such that $\epsilon+\delta<\frac{1}{4}$ and find a good base $(\beta, \tilde{B}, B)$ for $(\mathcal{U}, n, \epsilon, \delta)$. (Now we take $\tilde{B}$ to be a base for a strong Rohlin tower of height $N$ and error $<\delta$ and not of height $N+1$ as before). Set $N=\mathcal{N}\left(\mathcal{U}_{0}^{n-1}, \epsilon\right)$, so $B$ is the union of $N$ elements of $\left.\beta\right|_{\tilde{B}}$. We index these elements by sequences $i_{0} \ldots i_{n-1}$, such that if $B_{i_{0} \ldots i_{n-1}}$ is one, then $T^{j}\left(B_{i_{0} \ldots i_{n-1}}\right) \subset U_{i_{j}}$, for every $0 \leq j \leq n-1$. We have that $\mu\left(X \backslash \bigcup_{0}^{n-1} T^{i}(B)\right) \leq \epsilon+\delta$. Let $\hat{\alpha}=\left\{\hat{A}_{1} \ldots \hat{A}_{M}\right\}$ be the partition of

$$
E=\bigcup_{0}^{n-1} T^{i}(B)
$$

defined by

$$
\hat{A}_{m}=\bigcup\left\{T^{j}\left(B_{i_{0} \ldots i_{n-1}}\right) \mid j \in[0, n-1] . i_{j}=m\right\} .
$$

Note that $\hat{A}_{m} \subset U_{m}$, for every $1 \leq m \leq M$. Extend $\hat{\alpha}$, to a partition, $\alpha$, of $X$, refining $\mathcal{U}$, in some way. Set $\eta^{2}=\epsilon+\delta$ and define for every $k>n f_{k}(x)=\frac{1}{k} \sum_{o}^{k-1} \chi_{E}\left(T^{j} x\right)$. We have that $0 \leq f_{k} \leq 1$ and $\int f_{k}>1-\eta^{2}$, so if we will denote:

$$
G_{k}=\left\{x \mid f_{k}(x)>1-\eta\right\}
$$

then,

$$
\begin{aligned}
\eta \cdot \mu\left(G_{k}^{c}\right) \leq & \int_{G_{k}^{c}} 1-f_{k} \leq \int 1-f_{k} \leq \eta^{2} \\
& \Rightarrow \mu\left(G_{k}\right) \geq 1-\eta .
\end{aligned}
$$

We shall show that we can cover $G_{k}$, by "few" $(\alpha,[0, k-1])$-names. Partition $G_{k}$ according to the values of $0 \leq i \leq k-n$, such that $T^{i} x \in B$. Note that if $x \in G_{k}$ and $0 \leq i_{1}<$ $\cdots<i_{m} \leq k-n$, are the times in which $x$ visits $B$, then the collection $\left\{\left[i_{j}, i_{j}+n-1\right]\right\}_{j=1}^{m}$ covers all but at most $\eta k+2 n$ elements of $[0, k-1]$. Because each element of this partition defines a collection of subintervals of $[0, k-1]$, of length $n$, that covers all but at most 
$\eta k+2 n$, elements of $[0, k-1]$, in a $1-1$ manner, we have that the number of elements in the partition of $G_{k}$ is at most

$$
\psi(k, n, \eta)=\sum_{j<\left(\eta+\frac{2 n}{k}\right) k}\left(\begin{array}{l}
k \\
j
\end{array}\right)
$$

We fix an element $C$ of this partition of $G_{k}$ and want to estimate the number of $(\alpha,[0, k-$ 1])-names, needed to cover it. If $0 \leq i_{1}<\cdots<i_{m} \leq k-n$ are the times elements of $C$ visit $B$, then we need at most $N,\left(\alpha,\left[i_{j}, i_{j}+n-1\right]\right)$-names, to cover $C$. Because the size of $[0, k-1] \backslash \bigcup_{j}\left[i_{j}, i_{j}+n-1\right]$, is at most $\eta k+2 n$, we need at most $N^{\frac{k}{n}} \cdot M^{\eta k+2 n}$ $(\alpha,[0, k-1])$-names, to cover $C$. Finally, we have that we can cover $G_{k}$, by no more than:

$$
\psi(k, n, \eta) \cdot N^{\frac{k}{n}} \cdot M^{\eta k+2 n}
$$

$(\alpha,[0, k-1])$-names. Because $\mu\left(G_{k}\right)>1-\eta$, this means that:

$$
\frac{1}{k} \log \mathcal{N}\left(\alpha_{0}^{k-1}, \eta\right) \leq \frac{1}{k} \log \psi(k, n, \eta)+\frac{1}{n} \log N+\left(\eta+\frac{2 n}{k}\right) \log M .
$$

Recall that once $\left(\eta+\frac{2 n}{k}\right)<\frac{1}{2}$, we have $\psi(k, n, \eta) \leq 2^{k \cdot H\left(\eta+\frac{2 n}{k}\right)}$ and so

$$
h_{\mu}(\alpha)=\lim \frac{1}{k} \log \mathcal{N}\left(\alpha_{0}^{k-1}, \eta\right) \leq \frac{1}{n} \log \mathcal{N}\left(\mathcal{U}_{0}^{n-1}, \epsilon\right)+\eta \cdot \log M+H(\eta)
$$

SO

$$
h_{\mu}^{+}(\mathcal{U}) \leq \frac{1}{n} \log \mathcal{N}\left(\mathcal{U}_{0}^{n-1}, \epsilon\right)+\sqrt{\epsilon+\delta} \cdot \log M+H(\sqrt{\epsilon+\delta})
$$

Letting $\delta \rightarrow 0$ we get

$$
h_{\mu}^{+}(\mathcal{U}) \leq \frac{1}{n} \log \mathcal{N}\left(\mathcal{U}_{0}^{n-1}, \epsilon\right)+\sqrt{\epsilon} \cdot \log M+H(\sqrt{\epsilon})
$$

as desired.

4.5. Theorem. $h_{\mu}^{+}(\mathcal{U})=h_{\mu}^{-}(\mathcal{U})$

We already know that $h_{\mu}^{+}(\mathcal{U}) \geq h_{\mu}^{-}(\mathcal{U})$ (Proposition 3.6), so we only need to prove the other inequality. Before we turn to the proof, let us present some terminology and prove a combinatorial lemma.

Let $\Lambda$, be a finite alphabet of $M$ letters, $k, n \in \mathbb{N} k>>n, 0<\delta<1$ and $\omega=\omega_{0}^{k-1}$, a word of length $k$ on $\Lambda$. (The symbol $a_{r}^{s}$ stands for $a_{r} \ldots a_{s}$ ). Denote $\Gamma=\Lambda^{n}$.

- An $(n, k, \delta)$-packing is a pair $\mathcal{C}=\left(i_{0}^{m-1}, \gamma_{0}^{m-1}\right)$ where $0 \leq i_{j} \leq k-n, \gamma_{j} \in \Gamma, j=$ $0 \ldots m-1, i_{j}+n-1<i_{j+1}$ and $\frac{m \cdot n}{k}>1-\delta$. (We think of an $(n, k, \delta)$-packing as instructions to "almost" write a word of length $k$, we just fill it with the $\gamma_{j}$ 's, where $\gamma_{j}$ starts in the $i_{j}$ letter and there will be no more than $\delta k$ letters to add.)

- An $(n, k, \delta)$-packing for $\omega$, is an $(n, k, \delta)$-packing, $\mathcal{C}=\left(i_{0}^{m-1}, \gamma_{0}^{m-1}\right)$, such that $\omega_{i_{j}}^{i_{j}+n-1}=\gamma_{j}$. 
- if $\mu_{1}, \mu_{2}$ are probability distributions on $\Gamma$ then

$$
|| \mu_{1}-\mu_{2} \|=\max _{\gamma}\left|\mu_{1}(\gamma)-\mu_{2}(\gamma)\right|
$$

- An $(n, k, \delta)$-packing, $\mathcal{C}=\left(i_{0}^{m-1}, \gamma_{0}^{m-1}\right)$, induces a probability distribution on $\Gamma$, denoted by $P_{\mathcal{C}}$, by the formula $P_{\mathcal{C}}(\gamma)=\frac{1}{m} \#\left\{0 \leq j \leq m-1 \mid \gamma=\gamma_{j}\right\}$.

- If $\mu$ is a probability distribution on $\Gamma$ and $\mathcal{C}$ is an $(n, k, \delta)$-packing, then we say that $\mathcal{C}$ is $(n, k, \delta, \mu)$, if $\left\|\mu-P_{\mathcal{C}}\right\|<\delta$. We say that $\omega$ is $(n, k, \delta, \mu)$, if there is an $(n, k, \delta)$-packing for $\omega$, which is $(n, k, \delta, \mu)$.

4.6. Lemma. If $\mu$ is a probability distribution on $\Gamma$, with "average entropy"

$$
h_{0}=-\frac{1}{n} \sum_{\gamma \in \Gamma} \mu(\gamma) \log \mu(\gamma)
$$

then there exists a positive function $\varphi(\delta)$, such that $\varphi(\delta) \rightarrow 0$ as $\delta \rightarrow 0$ and such that if $0<\delta<\frac{1}{2}$, then for any $k>n$, the number of words $\omega \in \Lambda^{k}$, which are $(n, k, \delta, \mu)$, is at most $2^{k\left(h_{0}+\varphi(\delta)\right)}$.

Proof. Fix $k>n$. We want to estimate the number of words $\omega=\omega_{0}^{k-1} \in \Lambda^{k}$, that are $(n, k, \delta, \mu)$. For every such word, $\omega$, we can choose an $(n, k, \delta)$-packing, $\mathcal{C}=\left(i_{0}^{m-1}, \gamma_{0}^{m-1}\right)$ which is $(n, k, \delta, \mu)$. In this way we define a map

$$
\pi:\left\{\omega \in \Lambda^{k} \mid \omega \text { is }(n, k, \delta, \mu)\right\} \rightarrow\{\mathcal{C} \mid \mathcal{C} \text { is an }(n, k, \delta, \mu) \text { - packing }\}
$$

If $\mathcal{C}=\left(i_{0}^{m-1}, \gamma_{0}^{m-1}\right)$, is an $(n, k, \delta)$-packing, then $\frac{n \cdot m}{k}>1-\delta$. This means that $\left|\pi^{-1}(\mathcal{C})\right| \leq$ $|\Lambda|^{\delta k}=M^{\delta k}$. So we have that

$$
\#\left\{\omega \in \Lambda^{k} \mid \omega \text { is }(n, k, \delta, \mu)\right\} \leq M^{\delta k} \#\{\mathcal{C} \mid \mathcal{C} \text { is an }(n, k, \delta, \mu) \text { - packing }\} .
$$

Let us now estimate the number of $(n, k, \delta, \mu)$-packings, $\mathcal{C}=\left(i_{0}^{m-1}, \gamma_{0}^{m-1}\right)$ :

The number of sequences, $i_{0}^{m-1}$, such that $0 \leq i_{j} \leq k-n, i_{j}+n-1<i_{j+1}$ and $\frac{m \cdot n}{k}>1-\delta$ is at most $\sum_{j<\delta k}\left(\begin{array}{l}k \\ j\end{array}\right)$. From lemma 2.3 we know that for $\delta<\frac{1}{2}$, this sums to something $\leq 2^{H(\delta) k}$.

Fix such a sequence $i_{0}^{m-1}$. Let us now estimate the number of sequences, $\gamma_{0}^{m-1}$, such that the $(n, k, \delta)$-packing, $\mathcal{C}=\left(i_{0}^{m-1}, \gamma_{0}^{m-1}\right)$, is $(n, k, \delta, \mu)$.

Denote $\nu=\otimes_{1}^{m} \mu$, the product measure on $\Gamma^{m}$. If $\gamma_{0}^{m-1} \in \Gamma^{m}$, then

$$
\begin{gathered}
\nu\left(\gamma_{0}^{m-1}\right)=\prod_{\gamma \in \Gamma} \mu(\gamma)^{\#\left\{0 \leq j \leq m-1 \mid \gamma=\gamma_{j}\right\}}=2^{\sum_{\{\gamma \mid \mu(\gamma) \neq 0\}} \#\left\{0 \leq j \leq m-1 \mid \gamma=\gamma_{j}\right\} \cdot \log \mu(\gamma)} \\
=2^{m \sum_{\{\gamma \mid \mu(\gamma) \neq 0\}} \frac{1}{m} \#\left\{0 \leq j \leq m-1 \mid \gamma=\gamma_{j}\right\} \cdot \log \mu(\gamma)} .
\end{gathered}
$$

Now, the function $f:\left\{\left(x_{\gamma}\right)_{\gamma \in \Gamma} \in \mathbb{R}^{\Gamma} \mid \sum x_{\gamma}=1\right\} \rightarrow \mathbb{R}$, defined by

$$
f\left(\vec{x}_{\gamma}\right)=\sum_{\{\gamma \mid \mu(\gamma) \neq 0\}} x_{\gamma} \cdot \log \mu(\gamma)
$$


is continuous and so there is a positive function $\psi(\delta)$, such that $\psi(\delta) \rightarrow 0$ as $\delta \rightarrow 0$ and if $\max _{\gamma}\left|x_{\gamma}-\mu(\gamma)\right|<\delta$, then $\left|f\left(\vec{x}_{\gamma}\right)-f(\mu(\vec{\gamma}))\right|<\psi(\delta)$ (note that $\psi$ depends only on $n, \mu$ ). So if $\gamma_{0}^{m-1} \in \Gamma^{m}$ is such that $\mathcal{C}=\left(i_{0}^{m-1}, \gamma_{0}^{m-1}\right)$, is a $(n, k, \delta, \mu)$-packing, it follows that

$$
\begin{gathered}
\nu\left(\gamma_{0}^{m-1}\right)=2^{m \sum_{\{\gamma \mid \mu(\gamma) \neq 0\}} \frac{1}{m} \#\left\{0 \leq j \leq m-1 \mid \gamma=\gamma_{j}\right\} \cdot \log \mu(\gamma)} \\
\geq 2^{m\left(\sum_{\{\gamma \mid \mu(\gamma) \neq 0\}} \mu(\gamma) \log \mu(\gamma)-\psi(\delta)\right)} \geq 2^{k\left(-h_{0}-\frac{\psi(\delta)}{n}\right)}
\end{gathered}
$$

Where the last inequality follows from the fact that $m<\frac{k}{n}$ and the definition of $h_{0}$. We conclude that an upper bound for the number of such sequences $\gamma_{0}^{m-1}$ is $2^{k\left(h_{0}+\frac{\psi(\delta)}{n}\right)}$. If we collect these estimations, we get to the conclusion that for $0<\delta<\frac{1}{2}$

$$
\#\left\{\omega \in \Lambda^{k} \mid \omega i s(n, k, \delta, \mu)\right\} \leq M^{\delta k} \cdot 2^{H(\delta) k} \cdot 2^{k\left(h_{0}+\frac{\psi(\delta)}{n}\right)} \leq 2^{k\left(h_{0}+\frac{\psi(\delta)}{n}+H(\delta)+\delta \cdot \log M\right)}
$$

so $\varphi(\delta)=\frac{\psi(\delta)}{n}+H(\delta)+\delta \cdot \log M$ is our desired function.

Proof. (of theorem 4.5): We want to show that for an ergodic system $(X, \mathcal{B}, \mu, T)$ and a cover $\mathcal{U}=\left\{U_{1} \ldots U_{M}\right\}$ of $X$, we have $h_{\mu}^{+}(\mathcal{U}) \leq h_{\mu}^{-}(\mathcal{U})$. As before, if the system is periodic, then, from the ergodicity, it must be a cyclic permutation on a finite set of atoms. Therefore $h_{\mu}^{+}(\mathcal{U})=h_{\mu}^{-}(\mathcal{U})=0$. In the aperiodic case we can use the Strong Rohlin Lemma.

Let $\epsilon>0$. We shall show that $h_{\mu}^{+}(\mathcal{U}) \leq h_{\mu}^{-}(\mathcal{U})+2 \epsilon$. From the definition of $h_{\mu}^{-}(\mathcal{U})$, we can find $n \in \mathbb{N}$ and a partition $\beta \succeq \mathcal{U}_{0}^{n-1}$, such that $\frac{1}{n} H_{\mu}(\beta) \leq h_{\mu}^{-}(\mathcal{U})+\epsilon$. As $\beta \succeq \mathcal{U}_{0}^{n-1}$, we can index the elements of $\beta$, by sequences $i_{0}^{n-1}=i_{0} \ldots i_{n-1}$, such that if $\tilde{B}_{i_{0}^{n-1}}$, is one, then $T^{j} \tilde{B}_{i_{0}^{n-1}} \subset U_{i_{j}} j=0 \ldots n-1$. We can assume that each sequence, $i_{0}^{n-1}$, corresponds to, at most one element of $\beta$, for otherwise, we could unite these elements and get a coarser partition $\beta^{\prime}$, still refining $\mathcal{U}_{0}^{n-1}$, such that $\frac{1}{n} H_{\mu}\left(\beta^{\prime}\right) \leq \frac{1}{n} H_{\mu}(\beta) \leq h_{\mu}^{-}(\mathcal{U})+\epsilon$. Set $\Gamma=\{1 \ldots M\}^{n}$. So the elements of $\beta$ are indexed by $\Gamma$. (if $\gamma \in \Gamma$, does not correspond to an element of $\beta$, in the above way, we set $\left.\tilde{B}_{\gamma}=\emptyset\right)$. In this way, the partition $\beta$, defines a probability distribution, $\nu$, on $\Gamma$, defined by $\nu(\gamma)=\mu\left(\tilde{B}_{\gamma}\right)$ and we have that $h_{0}=\frac{1}{n} H_{\mu}(\beta)$, is the "average entropy" (see Lemma 4.6) of $\nu$.

Choose $\delta>0$ (in a manner specified later) and let $F$, be a base for a strong Rohlin tower (with respect to $\beta$ ) of height $n$ and error $\leq \delta^{2}$. Denote the atoms of $\left.\beta\right|_{F}$ by $B_{\gamma} \gamma \in \Gamma$, (where $B_{\gamma}=\tilde{B}_{\gamma} \cap F$ ), and define a partition $\tilde{\alpha}=\left\{\tilde{A}_{1} \ldots \tilde{A}_{M}\right\}$ of $E=\bigcup_{0}^{n-1} T^{j} F$, by $\tilde{A}_{m}=\cup\left\{T^{j} B_{i_{0}^{n-1}} \mid j \in\{0 \ldots n-1\}, i_{j}=m\right\}$. Note that $\tilde{A}_{m} \subset U_{m}$. Extend $\tilde{\alpha}$, to a partition $\alpha$ of $X$ refining $\mathcal{U}$, in some way. The set of indices of elements of $\alpha, \Lambda$ (the alphabet in which $\alpha$-names are written) contains $\{1 \ldots M\}$ and we can always build $\alpha$, such that $|\Lambda| \leq 2 M$. We slightly abuse our notation and denote $\Gamma=\Lambda^{n}$. In this way, $\nu$ is still a probability distribution on $\Gamma$.

Claim: If $\delta$, is small enough, then $h_{\mu}(\alpha) \leq h_{0}+\epsilon$.

Once we prove this claim, we are done, because then

$$
h_{\mu}^{+}(\mathcal{U}) \leq h_{\mu}(\alpha) \leq h_{0}+\epsilon \leq h_{\mu}^{-}(\mathcal{U})+2 \epsilon .
$$


Proof of claim: For $k>>n$, we look at the function $f_{k}(x)=\frac{1}{k} \sum_{0}^{k-1} \chi_{E}\left(T^{j} x\right)$. We have that $0 \leq f_{k} \leq 1$ and $\int f_{k}>1-\delta^{2}$. Therefore

$$
\begin{gathered}
\delta \cdot \mu\left(\left\{x \mid 1-f_{k}(x)>1-\delta\right\}\right) \leq \int_{\left\{x \mid 1-f_{k}(x)>1-\delta\right\}} 1-f_{k} \leq \int 1-f_{k} \leq \delta^{2} \\
\Rightarrow \mu\left(\left\{x \mid f_{k}(x) \geq 1-\delta\right\}\right) \geq 1-\delta .
\end{gathered}
$$

Denote, $G_{1}^{k}=\left\{x \mid f_{k}(x) \geq 1-\delta\right\}$. For $x \in G_{1}^{k}$, there are at most $\delta k$ times $0 \leq i \leq k-1$, such that $T^{i} x \notin E$. Define

$$
G_{2}^{k}=\left\{x|| \frac{1}{k} \sum_{0}^{k-n} \chi_{A}\left(T^{i} x\right)-\mu(A)|<\delta, A \in \beta|_{F} \cup\{F\}\right\} .
$$

Let us see what can we say about the $(\alpha,[0, k-1])$-name of an element, $x$, of $G_{1}^{k} \cap G_{2}^{k}$. Fix such an $x$ and denote by $i_{0}<\cdots<i_{m-1}$, the times between 0 to $k-n$ in which $x$ visits $F$. We have that $0 \leq i_{j} \leq k-n, i_{j}+n-1<i_{j+1}$ (that is because the height of the tower is $n$ ). Except for at most $2 n$ times ( $n$ at the beginning and $n$ at the end), $x$ visits $E$, exactly in the times $i_{j} \ldots i_{j}+n-1, j=1 \ldots m-1$. Therefore, we must have

$$
n \cdot m \geq(1-\delta) k-2 n \Rightarrow \frac{n \cdot m}{k} \geq 1-\left(\delta+\frac{2 n}{k}\right)
$$

Denote the $(\alpha,[0, k-1])$-name of $x$ by $\omega=\omega_{0}^{k-1}\left(\omega_{i} \in \Lambda\right)$, and $\gamma_{j}=\omega_{i_{j}} \ldots \omega_{i_{j}+n-1} \in \Gamma$, $j=0 \ldots m-1$. We have that $\mathcal{C}=\left(i_{0}^{m-1}, \gamma_{0}^{m-1}\right)$ is an $\left(n, k, \delta+\frac{2 n}{k}\right)$-packing for $\omega$. Let us now see, what can we say about the distribution, $P_{\mathcal{C}}$, this packing induces on $\Gamma$.

For $0 \leq r \leq k-n$, we have that $T^{r} x \in B_{\gamma}$ if and only if, there is a $0 \leq j \leq m-1$, such that $r=i_{j}$ and $\gamma=\gamma_{j}$. Therefore, because $x \in G_{2}^{k}$

- $\forall \gamma \in \Gamma \quad\left|\frac{1}{k} \#\left\{0 \leq j \leq m-1 \mid \gamma=\gamma_{j}\right\}-\mu\left(B_{\gamma}\right)\right|<\delta$.

- $\left|\frac{m}{k}-\mu(F)\right|<\delta$.

Note that $\mu(F)>\frac{1-\delta}{n}$, so if $\delta$ is sufficiently small, we can guarantee that $\left|\frac{k}{m}-\frac{1}{\mu(F)}\right|$ would be arbitrarily small and in turn we can guarantee that for every $\gamma \in \Gamma$

$$
\left|\frac{k}{m} \cdot \frac{1}{k} \#\left\{0 \leq j \leq m-1 \mid \gamma=\gamma_{j}\right\}-\frac{\mu\left(B_{\gamma}\right)}{\mu(F)}\right|=\left|P_{\mathcal{C}}(\gamma)-\nu(\gamma)\right|
$$

would be arbitrarily small. This is to say that $\left\|P_{\mathcal{C}}-\nu\right\|$ is arbitrarily small. We see that there is a positive function $\psi(\delta)$, independent of $k$, such that $\psi(\delta) \rightarrow 0$ as $\delta \rightarrow 0$ and such that, if $x \in G_{1}^{k} \cap G_{2}^{k}$ and $\omega$ is its $(\alpha,[0, k-1])$-name, then $\omega$ is $\left(n, k, \psi(\delta)+\frac{2 n}{k}, \nu\right)$.

Remember the function $\varphi$, from lemma 4.6. There is an $\eta_{0}>0$, such that for every $0<\eta<\eta_{0} \varphi(\eta)<\epsilon$. Choose $k$ to be large enough so that $\frac{2 n}{k}<\frac{\eta_{0}}{2}$ and the error, $\delta$, of the tower to be so small, such that $\psi(\delta)<\frac{\eta_{0}}{2}$, and conclude, from lemme 4.6, that the number of $(\alpha,[0, k-1])$-names of elements of $G_{1}^{k} \cap G_{2}^{k}$ is at most $2^{k\left(h_{0}+\epsilon\right)}$. From the ergodicity, we know that for large enough $k, \mu\left(G_{1}^{k} \cap G_{2}^{k}\right)>1-2 \delta$, so we have

$$
h_{\mu}(\alpha)=\lim \frac{1}{k} \log \mathcal{N}\left(\alpha_{0}^{k-1}, 2 \delta\right) \leq h_{0}+\epsilon .
$$

as desired. 
Remarks:

- If $(X, T)$, is totally ergodic, i.e $\left(X, T^{n}\right)$, is ergodic for every $n \in \mathbb{N}$, then we can look at expressions like $h_{\mu}^{e}\left(\mathcal{U}_{0}^{n-1}, T^{n}\right)$. It follows from the definition that $h_{\mu}^{e}(\mathcal{U}, T)=\frac{1}{n} h_{\mu}^{e}\left(\mathcal{U}_{0}^{n-1}, T^{n}\right)$. This enables us to prove the last theorem without any hard work done. We know from theorem 4.4, that $h_{\mu}^{e}(\mathcal{U}, T)=h_{\mu}^{+}(\mathcal{U}, T)$ and therefore $h_{\mu}^{+}(\mathcal{U}, T)=\frac{1}{n} h_{\mu}^{+}\left(\mathcal{U}_{0}^{n-1}, T^{n}\right)$. But then, proposition 3.6 (which is elementary), gives: $h_{\mu}^{-}(\mathcal{U}, T)=\lim \frac{1}{n} h_{\mu}^{+}\left(\mathcal{U}_{0}^{n-1}, T^{n}\right)=h_{\mu}^{+}(\mathcal{U}, T)$ and this gives the desired result.

- The definitions of $h_{\mu}^{+}(\mathcal{U}), h_{\mu}^{-}(\mathcal{U})$, were introduced in [R] and discussed also in [Ye], [HMRY]. There, a proof of their equality was given only in the case where $(X, T)$, is a t.d.s, and $\mathcal{U}$ is an open cover. The proof was based on a reduction to a uniquely ergodic case and then a use of a variational inequality, proved in [GW].

- The definition of $h_{\mu}^{e}(\mathcal{U})$ is new. This definition helps us to prove directly a slight generalization of the variational inequality ,proved in $[\mathrm{GW}]$ and mentioned above, to the non-topological case. (Theorem 6.1).

- The proofs of theorems 4.2, 4.4, 4.5 and lemma 4.6 are based on ideas of B.Weiss and E.Glasner

\section{ERgodic DECOMPOSITION FOR $h_{\mu}^{+}, h_{\mu}^{-}$}

5.1. Theorem. (Proposition 5 in [HMRY]): Let $\mathcal{U}=\left\{U_{1} \ldots U_{M}\right\}$, be a cover of $X$, and $\mu=\int \mu_{x} d \mu(x)$, the ergodic decomposition of $\mu$ with respect to $T$. Then

$$
h_{\mu}^{+}(\mathcal{U}, T)=\int h_{\mu_{x}}^{+}(\mathcal{U}, T) d \mu(x) \quad h_{\mu}^{-}(\mathcal{U}, T)=\int h_{\mu_{x}}^{-}(\mathcal{U}, T) d \mu(x)
$$

\subsection{Corollary. $h_{\mu}^{+}(\mathcal{U})=h_{\mu}^{-}(\mathcal{U})$}

Proof. It follows immediately from the above and the ergodic case (Theorem 4.5)

From now on we will denote the number $h_{\mu}^{+}(\mathcal{U}, T)=h_{\mu}^{-}(\mathcal{U}, T)\left(=h_{\mu}^{e}(\mathcal{U}, T)\right.$ in the ergodic case), simply by $h_{\mu}(\mathcal{U}, T)$ or $h_{\mu}(\mathcal{U})$ or $h(\mathcal{U})$, when no ambiguity can occur.

\section{VARiational RELATIONS}

As always, let $\mathcal{U}=\left\{U_{1} \ldots U_{M}\right\}$, be a cover of the m.t.d.s $(X, \mathcal{B}, \mu, T)$. We can define the "combinatorial entropy" of $\mathcal{U}$ as

$$
h_{c}(\mathcal{U}, T)=\lim _{n} \frac{1}{n} \log \mathcal{N}\left(\mathcal{U}_{0}^{n-1}\right)
$$

where, $\mathcal{N}(\mathcal{V})$, is the minimum number of elements of $\mathcal{V}$, needed to cover the whole space. Note that the sequence $\log \mathcal{N}\left(\mathcal{U}_{0}^{n-1}\right)$, is sub-additive, hence the limit exists. If $(X, T)$ is a t.d.s and $\mathcal{U}$ is an open cover then we denote $h_{\text {top }}(\mathcal{U}, T)=h_{c}(\mathcal{U}, T)$. 
The next theorem was proved in $[\mathrm{GW}$ for topological dynamical systems and measurable covers. We give here a simple proof for the non topological case that uses the definition of $h_{\mu}^{e}(\mathcal{U})$.

6.1. Theorem. $h_{\mu}(\mathcal{U}) \leq h_{c}(\mathcal{U})$.

Proof. First, if the system is ergodic, then $h_{\mu}(\mathcal{U})=\lim \frac{1}{n} \log \mathcal{N}\left(\mathcal{U}_{0}^{n-1}, \frac{1}{2}\right)$ and as $\mathcal{N}\left(\mathcal{U}_{0}^{n-1}, \frac{1}{2}\right) \leq$ $\mathcal{N}\left(\mathcal{U}_{0}^{n-1}\right)$, we have

$$
h_{\mu}(\mathcal{U}) \leq \lim \frac{1}{n} \log \mathcal{N}\left(\mathcal{U}_{0}^{n-1}\right)=h_{\text {top }}(\mathcal{U})
$$

as desired. In the non ergodic case, let $\mu=\int \mu_{x} d \mu(x)$, be the ergodic decomposition of $\mu$. By theorem $5.1, h_{\mu}(\mathcal{U})=\int h_{\mu_{x}}(\mathcal{U}) d \mu(x)$, so from the first part we see that $h_{\mu}(\mathcal{U}) \leq$ $h_{c}(\mathcal{U})$.

Remark: Another simple proof of the above, uses the definition of $h_{\mu}^{-}(\mathcal{U})$ :

$$
\begin{aligned}
& H_{\mu}\left(\mathcal{U}_{0}^{n-1}\right)=\inf _{\alpha \succeq \mathcal{U}_{0}^{n-1}} H_{\mu}(\alpha) \leq \inf _{\alpha \succeq \mathcal{U}_{0}^{n-1}} \log |\alpha| \leq \log \mathcal{N}\left(\mathcal{U}_{0}^{n-1}\right) \\
& \Rightarrow h_{\mu}(\mathcal{U})=\lim \frac{1}{n} H_{\mu}\left(\mathcal{U}_{0}^{n-1}\right) \leq \lim \frac{1}{n} \log \mathcal{N}\left(\mathcal{U}_{0}^{n-1}\right)=h_{c}(\mathcal{U}) .
\end{aligned}
$$

From this stage, until the end of this paper we assume that $(X, T)$, is a t.d.s. We denote by $\mathcal{M}_{T}(X)$, the set of $T$-invariant probability measures on $X$ and by $\mathcal{M}_{T}^{e}(X)$, the set of ergodic ones. Also $\mathcal{C}_{X}^{o}$, will denote the set of finite open covers of $X$.

In $[\mathrm{BGH}]$, the following theorem was proved:

6.2. Theorem. (Theorem 1 in $[B G H]):$ If $\mathcal{U} \in \mathcal{C}_{X}^{o}$, then there exists $\mu \in \mathcal{M}_{T}(X)$, such that $h_{\mu}(\mathcal{U}) \geq h_{\text {top }}(\mathcal{U})$.

In light of theorem 6.1 we have that for every $\mathcal{U} \in \mathcal{C}_{X}^{o}$, one can find a measure $\mu \in$ $\mathcal{M}_{T}(X)$, such that $h_{\mu}(\mathcal{U})=h_{\text {top }}(\mathcal{U})$. In fact theorem 7 in [HMRY] now becomes:

6.3. Corollary. for every $\mathcal{U} \in \mathcal{C}_{X}^{o}$, one can find a measure $\mu \in \mathcal{M}_{T}^{e}(X)$, such that $h_{\mu}(\mathcal{U})=h_{\text {top }}(\mathcal{U})$.

Proof. Choose $\mu \in \mathcal{M}_{T}(X)$, such that $h_{\mu}(\mathcal{U})=h_{\text {top }}(\mathcal{U})$, and let $\mu=\int \mu_{x} d \mu(x)$, be its ergodic decomposition. We know that

$$
h_{\text {top }}(\mathcal{U})=h_{\mu}(\mathcal{U})=\int h_{\mu_{x}}(\mathcal{U}) d \mu(x)
$$

and that $h_{\mu_{x}}(\mathcal{U}) \leq h_{\text {top }}(\mathcal{U})$. So we must have $h_{\mu_{x}}(\mathcal{U})=h_{\text {top }}(\mathcal{U})$ for $[\mu]$ a.e $x$.

We conclude from the above, the classical variational principle:

First we state a technical lemma, taken from [Ye].

6.4. Lemma. For any $\epsilon>0, \mu \in \mathcal{M}_{T}(X)$ and $\alpha=\left\{A_{1} \ldots A_{M}\right\} \in \mathcal{P}_{X}$, there exists an open cover $\mathcal{U} \in \mathcal{C}_{X}^{o}$, such that for every partition $\beta \succeq \mathcal{U}$ one has $H_{\mu}(\alpha \mid \beta)<\epsilon$.

6.5. Theorem. (The Variational Principle):

(a) For every $\mu \in \mathcal{M}_{T}(X), h_{\mu}(T) \leq h_{\text {top }}(T)$. 
(b) $\sup _{\mu \in \mathcal{M}_{T}^{e}(X)} h_{\mu}(T)=h_{\text {top }}(T)$.

Proof. To prove $(a)$, we first show that for each $\mu \in \mathcal{M}_{T}(X), h_{\mu}(T)=\sup _{\mathcal{U} \in \mathcal{C}_{X}^{o}} h_{\mu}(\mathcal{U}, T)$. If this is done, then from theorem 6.1, we get

$$
h_{\mu}(T) \leq \sup _{\mathcal{U} \in \mathcal{C}_{X}^{o}} h_{t o p}(\mathcal{U}, T)=h_{\text {top }}(T)
$$

It follows from the definition, that for any cover $\mathcal{U}$ of $X$, we have $h_{\mu}(\mathcal{U}, T) \leq h_{\mu}(T)$, so one inequality is clear. For the other inequality, fix a partition, $\alpha=\left\{A_{1} \ldots A_{M}\right\}$, of $X$ and $\epsilon>0$. We need to find an open cover, $\mathcal{U}$, of $X$, such that $h_{\mu}(\alpha, T) \leq h_{\mu}(\mathcal{U}, T)+\epsilon$. By the preceding lemma and from the fact that for any $\beta \in \mathcal{P}_{X}$ one has $h_{\mu}(\alpha) \leq h_{\mu}(\beta)+H(\alpha \mid \beta)$ we have $\mathcal{U} \in \mathcal{C}_{X}^{o}$, such that

$$
h_{\mu}(\mathcal{U}, T)=\inf _{\beta \succeq \mathcal{U}} h_{\mu}(\beta, T) \geq \inf _{\beta \succeq \mathcal{U}}\left(h_{\mu}(\alpha, T)-H_{\mu}(\alpha \mid \beta)\right) \geq h_{\mu}(\alpha, T)-\epsilon .
$$

To prove $(b)$, note that from (6.3) we know that for any $\mathcal{U} \in \mathcal{C}_{X}^{o}$, we can find $\mu \in \mathcal{M}_{T}^{e}(X)$, such that $h_{\mu}(\mathcal{U}, T)=h_{\text {top }}(\mathcal{U}, T)$. This gives us

$$
\sup _{\mu \in \mathcal{M}_{T}^{e}(X)} h_{\mu}(T) \geq h_{\text {top }}(\mathcal{U}, T) \Rightarrow \sup _{\mu \in \mathcal{M}_{T}^{e}(X)} h_{\mu}(T) \geq \sup _{\mathcal{U} \in \mathcal{C}_{X}^{o}} h_{\text {top }}(\mathcal{U}, T)=h_{\text {top }}(T) .
$$

Together with $(a)$, we get equality, which is $(b)$.

\section{REFERENCES}

[BGH] F.Blanchard, E.Glasner and B.host. A variation on the variational principle and applications to entropy pairs. Ergod.th.ED Dynam.Sys. 17(1997),29-43.

[GW] E.Glasner and B.Weiss. On the interplay between measurable and topological dynamics. Preprint 2004.

[HMRY] W.Huang, A.Maass, P.P.Romagnoly and X.Ye. Entropy pairs and local abramov formula for measure-theoretic entropy for a cover. Ergod. Th. and Dynam. Sys.24(2004), 1127-1153.

[R] P.P.Romagnoli. A local variational principle for the topological entropy. Ergod.th.E Dynam.Sys.23(2003),1601-1610.

[Ru] D.J.Rudolph. Fundamentals of measurable dynamics: Ergodic Theory on Lebesgue Spaces. Oxford Clarendon Press, 1990.

[Sh1] P.C.Shields. The Ergodic Theory Of Discrete Sample Paths. AMS Graduate Studies in Mathematics vol.13. 1996.

[Sh2] P.C.Shields. The Theory of Bernoulli Shifts. Reprint of 1973 University of Chicago Press edition (available on the Web).

[Ye] W.huang, X.Ye and G.Zhang. A local variational principle for conditional entropy. Preprint 2004. 